\title{
Presence of Obstetrics Cholestasis in Mothers Presenting with Pruritus in Pregnancy: In a Low Resource South Asian Setting
}

\author{
Senani Hemantha Dodampahala1*, Hemantha Pieris ${ }^{2}$, Lal Gotabaya Chandrasena ${ }^{3}$, \\ Surangi Jayakody ${ }^{4}$, Chamara Gunathilaka5, Chandrika Neelakanthi Wijayaratne ${ }^{1}$, \\ Gayani Sandamali Kotuwegedara Dodampahala', Amila Ruwan Meegahawatta1 \\ ${ }^{1}$ University Gynaecology and Obstetrics Unit, De Zoysa Maternity Hospital, Colombo, Sri Lanka \\ ${ }^{2}$ University of Sri Jayewardenepura, Nugegoda, Sri Lanka \\ ${ }^{3}$ Faculty of Medicine, University of Kelaniya, Kelaniya, Sri Lanka \\ ${ }^{4}$ Post Graduate (MD) Trainee in Community Medicine, Colombo, Sri Lanka \\ ${ }^{5}$ De Soyza Maternity Hospital, Colombo, Sri Lanka \\ ${ }^{6}$ National Hospital of Sri Lanka, Colombo, Sri Lanka \\ Email: *hemanthadodampahala@yahoo.co.uk,hempeiris@yahoo.com,dgmnh@sltnet.lk, \\ surangijayakody@yahoo.com, C.gunathilaka@yahoo.com,mandika59@hotmail.com, hemdodam@gmail.com, \\ amilameegahawatta@gmail.com
}

Received 26 March 2016; accepted 6 May 2016; published 9 May 2016

Copyright (C) 2016 by authors and Scientific Research Publishing Inc.

This work is licensed under the Creative Commons Attribution-NonCommercial International License (CC BY-NC).

http://creativecommons.org/licenses/by-nc/4.0/

(c) () (9) Open Access

\section{Abstract}

Obstetric cholestasis (also referred to as intrahepatic cholestasis of pregnancy-ICP) is a pruritic form of reversible cholestasis that is associated with significant fetal risks. There is a paucity of research regarding pregnancy outcomes of the mothers with obstetric cholestasis in the South Asian setting. Hence, the objective of this study was to determine the prevalence of obstetric cholestasis among mothers presenting with pruritus during pregnancy and to describe the characteristics and outcomes for those diagnosed with ICP in comparison with those with pruritus in the absence of ICP. Methods: All mothers presenting with pruritus to De Soyza Maternity hospital in Colombo Sri Lanka, between 1st January 2011 to 1st January 2014, were recruited for the study. A standard set of biochemical tests were used for diagnosis of ICP. An interviewer administered questionnaire and patient records were used for data collection. Presentation, characteristics and pregnancy outcomes were assessed and compared for mothers with ICP against those with pruri-

\footnotetext{
"Corresponding author.
}

How to cite this paper: Dodampahala, S.H., et al. (2016) Presence of Obstetrics Cholestasis in Mothers Presenting with Pruritus in Pregnancy: In a Low Resource South Asian Setting. Advances in Reproductive Sciences, 4, 37-45. 
tus in the absence of ICP. Results: The prevalence of ICP in the study population was found to be $27 \%$ $(n=27)$. Generalized pruritus with pruritus of the palms and soles was identified as the commonest pattern of pruritus for diagnosed with ICP. Delivery before 37 weeks $(p=0.001)$, meconium stained liquor $(p=0.004)$, placental abruption $(p=0.005)$, postpartum hemorrhage $(p=0.005)$, bleeding manifestation $(p=0.006)$, preterm labor $(p=0.031)$ and fetal distress $(p=0.035)$ were found to be significantly associated with the presence of ICP. Conclusions: Nearly $1 / 3$ of mothers presenting with pruritus in pregnancy in Sri Lanka are affected by ICP which is associated with an increase in a wide range of adverse maternal and fetal outcomes compared to those with pruritus in the absence of ICP.

\section{Keywords}

\section{Bile Acid, Intrahepatic Cholestasis, Liver Disease, Pregnancy Complications, Pruritus}

\section{Introduction}

Obstetric cholestasis (also referred to as intrahepatic cholestasis of pregnancy) is a liver disease unique to pregnancy characterized by pruritus in the absence of a skin rash with abnormal liver function tests; which occurs in the second or third trimesters and disappears spontaneously after delivery. The clinical importance of obstetric cholestasis lies in the potential fetal risks, which may include spontaneous preterm birth, iatrogenic preterm birth and fetal death. There can also be maternal morbidity in association with the intense pruritus and consequent sleep deprivation [1]. Routine liver function tests show raised transaminases in $60 \%$ of patients and raised bilirubin concentrations in only $25 \%$. Serum bile acids are raised in the vast majority of patients [2].

A genetic background is suggested by family clustering and demographic variations with the highest incidences reported from Chile-Bolivia (6\% - 27\%) and lowest incidence in England (0.7\%) [3]. ICP is associated with an increased risk of preterm delivery in $19 \%$ - 60\% [4], intra-partum fetal distress in $22 \%-41 \%$, and intrauterine fetal death (IUFD) in $0.75 \%-1.6 \%$ of the affected pregnancies [5]. The diagnostic criteria for ICP have varied over time in different reports, making complication rates difficult to compare. When, in addition to pruritus, clinical jaundice was used to define ICP. Higher fetal complication rates were reported than when diagnosis was based only on elevated bile acid and transaminase levels [6]. The Swedish ICP incidence figure is taken from a study using only pruritus in pregnancy as the inclusion criterion, and hence did not report increased fetal risk associated with ICP.

The management of intrahepatic cholestasis in pregnancy is overshadowed by the increased risks of fetal distress, spontaneous preterm delivery and fetal death [7]. The causes of these are unknown but are thought to be due to abnormal placental vascular response to elevated maternal serum bile acids [6]. Evidence from animal studies have suggested that elevated serum bile acids impair the trophoblastic microvascular circulation, hence impeding the placental transfer [8]. Risks are unrelated to the severity of symptoms and increase near term. Close monitoring of fetal wellbeing is essential, although no single test reliably predicts the risk of intrauterine death [9]. Raised serum concentrations of maternal bile acids correlate with the severity of pruritus and risk of fetal distress [8]. Some obstetricians recommend regular non-stress and contraction stress tests. According to Green-top guidelines by RCOG, delivery should ideally take place around 38 weeks after serial estimations of fetal lung maturity and maternal serum concentrations of total bile acid and delivery around 36 weeks should be considered for severe intrahepatic cholestasis of pregnancy with jaundice and progressive increases in serum bile acid concentrations if fetal distress is suspected and lung maturity has been confirmed.

\section{Justification}

There is paucity of South Asian data on intrahepatic cholestasis of pregnancy and maternal and fetal outcomes. Identification of mothers with obstetric cholestasis and proper management adhering to guidelines will invariably reduce the fetal morbidity and mortality as well as maternal morbidity. Therefore a hospital based longitudinal study was designed to determine the prevalence of obstetric cholestasis among mothers presenting with pruritus during pregnancy and to describe the characteristics and outcomes for those diagnosed with ICP in 
comparison with those with pruritus in the absence of ICP.

\section{Materials and Methods}

Study design: Hospital based longitudinal study.

Study setting: The study was conducted in De Soyza Hospital for women in Colombo, which is a tertiary care referral center for obstetric and gynecology patients in the state health sector for medically complicated pregnancies in Sri Lanka. It provides dedicated service for treating complicated antenatal mothers through multidisciplinary inputs over the past two decades. Around 5000 deliveries take place per year in this center.

Study period: The study was conducted from 1t January 2011 to $31^{\text {st }}$ December 2014.

\section{Sample size calculation:}

The following formula is used to calculate sample size (Lwanga and Lemeshow, 1991);

$$
\mathrm{n}=\frac{\mathrm{Z}^{2} \times \mathrm{P}(1-\mathrm{P})}{\mathrm{d}^{2}}
$$

n-total sample size.

$\mathrm{P}$ - expected population proportion.

$\mathrm{P}=6 \%$ (prevalence of obstetric cholestasis in Chile-Bolivia 6\% [3]).

$\mathrm{Z}=1.96$ (critical value for $95 \%$ confidence interval).

$\mathrm{d}=0.05$ (accepted amount of absolute error is considered as $5 \%$ ).

Therefore,

$$
\begin{gathered}
\mathrm{n}=\frac{1.962 \times 0.06(1-0.06)}{(0.05)^{2}} \\
\mathrm{n}=\frac{3.8416 \times 0.06 \times 0.94}{0.0025} \\
\mathrm{n}=86
\end{gathered}
$$

To compensate for non-respondents, $10 \%$ of the calculated sample size is added making total sample size as 97.

Study population: There were 100 pregnant women with pruritus who fulfill eligibility criteria during the study period in the above referral center. As the required sample size was 97, all were recruited after obtaining informed written consent.

Mothers with preexisting liver or renal disorders, systemic allergy or dermatological conditions that could result in pruritus were excluded from the study.

\section{Data Collection}

The purpose of the study was explained to the pregnant women and informed written consent was obtained. Pre tested, interviewer-administered questionnaire were used for data collection. Blood was taken for biochemical assay of serum and urinary bile acids and liver function tests viz. SGOT, SGPT, ALP, bilirubin (direct \& indirect) serum protein PT/INR.

Data was obtained from the study population both retrospectively and prospectively by medical graduates not directly involved in patient management through a questionnaire based interview and study of clinic and patient records. Collected data included socio-demographics, past medical and obstetric histories, present obstetric history, onset/associated features of pruritus, antenatal complications and the treatment given.

Clinical presentation and obstetric outcome were measured along with the type of delivery, period of gestation at delivery, weight for gestational age, and maternal and neonatal complications following delivery. Maternal co-morbidities together with investigations were also recorded.

\section{Statistical Analysis}

Statistical Package for Social Sciences (SPSS) version 17 for Microsoft Windows was used for statistical analysis. In the first step, patients were categorized according to serum bile acid levels: "no ICP” (bile acid levels 10 
$\mu \mathrm{mol} / \mathrm{L}$ ), "mild ICP" (bile acid levels of 10 - $39 \mu \mathrm{mol} / \mathrm{L}$ ), and "severe ICP" (bile acid levels $\geq 40 \mu \mathrm{mol} / \mathrm{L}$ any time) [6]. The outcome measures; maternal and fetal complications, time and mode of delivery, birth weight were compared between the two groups (ICP present and absent) using the Chi Square test. $\mathrm{P}$ value $<0.05$ was considered statistically significant.

\section{Ethical Approval}

Ethical clearance was obtained by the Ethics Review Committee, Faculty of Medical Sciences, University of Sri Jayewardenepura Sri Lanka.

\section{Results}

Hundred mothers aged between 19 - 39 years, presented with pruritus to the two hospital clinics during the study period and were recruited for the survey after obtaining informed written consent. The mean age of the study population was 29 years and over $80 \%$ of the study population was aged between 21 and 35 years. A majority ( $\mathrm{n}=$ 58) $58 \%$ had developed pruritus after 28 weeks of gestation while only a few $(n=8,8 \%)$ had developed jaundice at any time during pregnancy. Vomiting $(23 \%, \mathrm{n}=23)$ and abdominal pain $(21 \%, \mathrm{n}=21)$ were the commonest complains in addition to pruritus. Seventeen (17\%) mothers had been diagnosed with gestational diabetes mellitus (GDM) while 11 (11\%) were diagnosed with pregnancy induced hypertension (PIH). From the time of recruiting, the mothers were followed up until the end of their pregnancy. No neonatal deaths occurred within the study population. Sixty five (65) mothers delivered by normal vaginal delivery (NVD) while there were 16 elective cesarean sections (El-CS) and 19 emergency cesarean sections (Em-CS). Table 1 summarizes the characteristics of the study population.

Serum bile acid levels were tested for each study participant to determine the presence and the severity of intrahepatic cholestasis of pregnancy (ICP). Only 27 (27\%) mothers were found to have serum bile acids levels of $10 \mu \mathrm{mol} / \mathrm{L}$ and above indicating the presence of ICP [6]. All patients with increased serum bile acid levels belonged to the category of mild intrahepatic cholestasis (serum bile acids between $10-39 \mu \mathrm{mol} / \mathrm{L}$ ). The mean serum bile acid level for those with ICP was $17.7 \mu \mathrm{mol} / \mathrm{L}$. Out of these $20(74 \%)$ were found to have elevated SGOT (AST) and 17 (64.7\%) were found to have elevated SGPT (ALT).

The characteristics of the 27 mothers with mild ICP was compared with the rest of the study population and

Table 1. Characteristics of the study population.

\begin{tabular}{|c|c|}
\hline Characteristics & Total (\%) \\
\hline \multicolumn{2}{|c|}{ Age distribution } \\
\hline$<20$ & $4(4 \%)$ \\
\hline $21-35$ & $81(81 \%)$ \\
\hline$>35$ & $15(15)$ \\
\hline \multicolumn{2}{|c|}{ Development of pruritus } \\
\hline$<28$ weeks & $42(42 \%)$ \\
\hline After 28 weeks & $58(58 \%)$ \\
\hline \multicolumn{2}{|c|}{ Presence of diseases complicating pregnancy } \\
\hline Gestational diabetes mellitus & $17(17 \%)$ \\
\hline Pregnancy induced hypertension & $11(11 \%)$ \\
\hline \multicolumn{2}{|c|}{ Mode of delivery } \\
\hline Normal vaginal & $65(65 \%)$ \\
\hline Elective cesarean section & $16(16 \%)$ \\
\hline Emergency cesarean section & 19 (19\%) \\
\hline
\end{tabular}


look for the presence of significantly associated characteristics. Nausea was found to be significantly associated ( $p=0.0001)$ with the presence of ICP in mother with pruritus. It was present in 22 of the $27(82.4 \%)$ mothers diagnosed with ICP while only 18 out of the remaining 73 (25.7\%) complained of nausea. Commonest pattern of pruritus in those with ICP was found to be generalized pruritus with pruritus of the palms and soles $(\mathrm{n}=19$, 70.6\%) while generalized pruritus alone was the commonest pattern (47.1\%) in those without elevated serum bile acids. These differences in pruritus pattern were found to be significant with a Pearson Chi square value of 0.024. Age $(p=0.588)$, BMI $(p=0.060)$ and presence or absence of jaundice $(p=0.060)$, presence of GDM $(p=$ $0.075)$ or PIH ( $\mathrm{p}=0.34)$ did not vary significantly between those with ICP and those with pruritus alone.

The study group was followed up until delivery and their pregnancy outcomes as well as complications were noted. The maternal and fetal outcomes of mothers with ICP were compared with those without ICP. Overall, there were a total of 19 emergency cesarean sections for the study population, with 12 of them carried out on mothers diagnosed with ICP indicating that mother with ICP were over 2 times more likely to need emergency cesarean section.

Only 6 (23.5\%) of the mothers diagnosed with ICP had pregnancy extending beyond 37 weeks of gestation compared to the 56 (77.1\%) who did so in the non ICP group. Most (17) of the deliveries in the ICP group occurred between 33-37 weeks of gestation. Statistical analysis revealed that differences in POA (0.001) including POA of less than 37 weeks at the time of delivery as well as the presence of pre-term labor $(p=0.031)$ were significantly associated with the presence of ICP among mothers with pruritus. Meconium stained liquor was the commonest complication ( $\mathrm{n}=19,70.6 \%)$ noted in mothers with ICP followed the presence of fetal distress $(\mathrm{n}=$ 16, 58.8\%). Eight of the mothers also had bleeding manifestations out of which a majority were $(n=6,75 \%)$ mothers diagnosed with ICP. All three of meconium stained liquor, fetal distress and bleeding manifestations were found to be significantly associated with the presence of ICP complicating pregnancy compared to the control group. Six of the patients (22\%) with ICP suffered placental abruption during pregnancy as opposed to just 2 (2.7\%) from the non ICP group. Presence of placental abruption also significantly associated with ICP ( $\mathrm{p}=$ 0.005). Similar numbers were observed for the occurrence of postpartum hemorrhage (PPH) with 8 out of the 10 pregnancies complicated with PPH belonging to the group diagnosed with ICP, corresponding to a Pearson Chi-square value of 0.005 . Interestingly, we failed to find similar correlations between the presence of abnormal Doppler ultrasound $(p=0.343)$ findings, fetal abnormality $(p=0.488)$ or IUGR $(p=0.106)$ and ICP. Table 2 summarizes the complications significantly associated with the presence of ICP. Table 3 summarizes the characteristics of the patients with ICP.

\section{Discussion}

The above study was conducted in a most advanced maternity care hospital in the state healthcare sector both in the western province as well as in the country. For this reason, the rate of pregnancy complications in mothers treated at these units tends to be higher than in other parts of the country.

We targeted the study towards mother presenting with pruritus in order to make sure those with the highest risk of ICP are screened for its presence. The biochemical screening was carried out in the private sector due to absence of facilities for such testing in the state sector hospitals. Our finding suggested that upto $1 / 3$ of the mothers presenting with pruritus in pregnancy may be affected by ICP.

All patient with ICP in our study were categorized as having mild cholestasis on the basis of serum bile acid

Table 2. Complication significantly associated with the presence of ICP.

\begin{tabular}{cccc}
\hline Outcome & $\begin{array}{c}\text { Number with ICP } \\
(\mathbf{n}=\mathbf{2 7})\end{array}$ & $\begin{array}{c}\text { Number without ICP } \\
\text { (n = 73) }\end{array}$ & $17(22.9 \%)$ \\
pelivery before 37 weeks & $21(76.47)$ & $10(28.6 \%)$ & 0.001 \\
Meconium stained liquor & $19(70.6 \%)$ & $2(5.7 \%)$ & 0.004 \\
Bleeding Manifestations & $6(35.3 \%)$ & $10(13.7 \%)$ & 0.006 \\
Fetal distress & $16(59.2 \%)$ & $2(2.9 \%)$ & 0.035 \\
Placental abruption & $6(22.2 \%)$ & $2(2.9 \%)$ \\
\hline
\end{tabular}


levels. The absence of patients with moderate and severe ICP is likely attributable to the limited size of the study population. The average serum bile acid for those with ICP was $17.7 \mu \mathrm{mol} / \mathrm{L}$, which was lower than those observed for patient with mild ICP in other studies. However, notably the need for cesarean section, both elective and emergency (70.6\%), were higher [10] [11]. Reasons for this possibly is due to fetal distress [12]. Overall mothers with ICP were nearly 5 times more likely to deliver by cesarean section [7]. Evidence suggest that delivery of

Table 3. Details of the 27 patients diagnosed with ICP.

\begin{tabular}{|c|c|c|c|c|c|c|c|c|c|c|c|}
\hline Index no. & Age & Parity & BMI & $\begin{array}{l}\text { Onset of } \\
\text { pruritus }\end{array}$ & Site & $\begin{array}{c}\text { Se. bile } \\
\text { acid level } \\
\mu \mathrm{mol} / \mathrm{L}\end{array}$ & $\begin{array}{c}\text { Drug } \\
\text { treatment }\end{array}$ & $\begin{array}{c}\text { Time of } \\
\text { delivery } \\
\text { (in weeks) }\end{array}$ & Mode & $\begin{array}{c}\text { Fetal } \\
\text { outcome }\end{array}$ & $\begin{array}{c}\text { Birth } \\
\text { wt.(kg) }\end{array}$ \\
\hline 1 & 35 & 1 & 27 & 32 & $\mathrm{p} / \mathrm{s}$ & 13.60 & Antihist. & 38 & CS & IUGR & 2.80 \\
\hline 2 & 36 & 2 & 30 & 30 & G & 24.70 & UDCA & 36 & EmCS & $\begin{array}{l}\text { FD } \\
\text { IUGR }\end{array}$ & 2.43 \\
\hline 3 & 38 & 2 & 30 & 32 & G & 13.00 & UDCA & 39 & VD & NAD & 2.98 \\
\hline 4 & 28 & 3 & 32 & 24 & G & 27.40 & UDCA & 34 & CS & $\begin{array}{l}\text { FD } \\
\text { IUGR }\end{array}$ & 2.10 \\
\hline 5 & 31 & 2 & 21 & 30 & $\mathrm{p} / \mathrm{s}$ & 22.10 & UDCA & 36 & EmCS & FD & 2.80 \\
\hline 6 & 23 & 1 & 27 & 29 & G & 11.40 & UDCA & 31 & EmCS & $\begin{array}{l}\text { FD } \\
\text { IUGR }\end{array}$ & 2.20 \\
\hline 7 & 31 & 3 & 31 & 28 & G & 24.60 & UDCA & 34 & EmCS & FD & 2.42 \\
\hline 8 & 33 & 1 & 23 & 22 & $\mathrm{p} / \mathrm{s}$ & 12.80 & Antihist. & 37 & VD & IUGR & 2.30 \\
\hline 9 & 32 & 2 & 28 & 24 & G & 20.00 & UDCA & 37 & CS & FD & 3.20 \\
\hline 10 & 33 & 4 & 30 & 27 & G & 12.60 & UDCA & 38 & VD & FD & 2.70 \\
\hline 11 & 38 & 1 & 31 & 31 & G & 24.70 & UDCA & 37 & EmCS & FD & 2.80 \\
\hline 12 & 32 & 2 & 28 & 30 & G & 22.90 & UDCA & 36 & EmCS & $\begin{array}{l}\text { FD } \\
\text { IUGR }\end{array}$ & 2.10 \\
\hline 13 & 31 & 2 & 20 & 27 & G & 11.50 & UDCA & 36 & VD & FD & 2.60 \\
\hline 14 & 32 & 3 & 27 & 27 & $\mathrm{p} / \mathrm{s}$ & 12.80 & Antihist. & 38 & VD & NAD & 2.90 \\
\hline 15 & 25 & 1 & 25 & 28 & G & 11.90 & Antihist. & 37 & CS & NAD & 2.78 \\
\hline 16 & 32 & 4 & 22 & 28 & G & 12.90 & UDCA & 35 & EmCS & FD & 2.50 \\
\hline 17 & 32 & 2 & 30 & 30 & G & 22.00 & UDCA & 34 & CS & $\begin{array}{l}\text { FD } \\
\text { IUGR }\end{array}$ & 2.20 \\
\hline 18 & 33 & 4 & 30 & 32 & G & 12.60 & UDCA & 38 & VD & FD & 2.70 \\
\hline 19 & 38 & 1 & 31 & 24 & G & 24.70 & UDCA & 37 & EmCS & FD & 2.80 \\
\hline 20 & 32 & 2 & 28 & 26 & G & 22.90 & UDCA & 36 & EmCS & FD, IUGR & 2.10 \\
\hline 21 & 31 & 2 & 20 & 28 & $\mathrm{p} / \mathrm{s}$ & 11.50 & UDCA & 36 & VD & FD & 2.60 \\
\hline 22 & 32 & 3 & 27 & 30 & G & 12.80 & Antihist. & 38 & VD & NAD & 2.90 \\
\hline 23 & 25 & 1 & 26 & 31 & $\mathrm{p} / \mathrm{s}$ & 11.90 & Antihist. & 37 & CS & NAD & 2.70 \\
\hline 24 & 31 & 4 & 28 & 30 & g & 12.90 & UDCA & 35 & EmCS & FD & 2.50 \\
\hline 25 & 32 & 2 & 30 & 32 & G & 22.00 & UDCA & 34 & EmCS & FD, IUGR & 2.20 \\
\hline 26 & 28 & 2 & 24 & 30 & $\mathrm{p} / \mathrm{s}$ & 21.60 & UDCA & 36 & VD & NAD & 2.45 \\
\hline 27 & 30 & 1 & 27 & 25 & G & 15.20 & Antihis. & 31 & CS & IUGR & 1.90 \\
\hline
\end{tabular}

Abbreviations: p/s_palms and soles, G-generalized, Antihis: Antihistamines, UDCA: Ursodeoxycholic acid, CS—Cesarean section, Em.CS: Emergency Cesarean section, VD: Vaginal delivery, IUGR: Intrauterine growth retardation, FD—Fetal distress, NAD—no abnormalities detected. 
mother with ICP should be ideally carried out between 36 - 38 weeks of gestation with new evidence suggesting an increased risk of neonatal complications for every week the pregnancy continued beyond 36 weeks [1] [13] [14]. In our own study population, only 6 mothers had pregnancy continued beyond 37 weeks of gestation. This likely explains the observation of no still births or neonatal deaths in the study population. However, the high level of emergency cesarean sections (59\%) indicates that delivery period was often a result of maternal or neonatal complications.

Despite the absence of fetal or neonatal deaths, significantly higher incidence of complications was observed in mothers diagnosed with ICP compared to the rest of the population. The increase in complications noted in the study correlate well with the findings of similar studies [2] [3] [15]-[17]. Postpartum hemorrhage and placental abruption showed the greatest increase in those with ICP, with roughly a 10 fold increase over those who had pruritus in the absence of ICP [18]. The Increase in bleeding manifestations such as PPH is well recognized [15] [19] and is thought to occur as a result of subclinical steatorrhea associated with ICP resulting in reduced absorption of vitamin K [20] [21]. Fetal distress, another complication known to be associated with ICP was twice more common among mothers with ICP in our study. Reasons for fetal distress and placental abruption as well many of the other adverse effects are thought be the effects of bile acids on the placenta. Studies have indicated that bile acids can cause placenta vasoconstriction [22] and affect the placental transport and hormone production. In addition bile acids have been shown to increase myometrial sensitivity to oxytocin in humans and cause colonic contraction in animal fetuses increasing the passage of meconium [18]. These findings likely explain the roughly 3 fold increase in meconium stained liquor and pre-term labor observed among the mothers with ICP. Interestingly no significant in Doppler ultrasound finding or IUGR was noted among mother with ICP. This can be explained by the findings that suggest neonatal complications observed in ICP are not due to chronic placental insufficiency which can be detected by Doppler ultrasound and causes IUGR [23].

\section{Conclusion}

Our findings suggest that about $1 / 3$ of the mothers presenting with pruritus in pregnancy in Sri Lanka may be affected by ICP. Mothers with ICP in Sri Lanka demonstrated a significant increase in maternal and fetal complications consistent with findings in similar studies elsewhere.

\section{Recommendations}

We recommend that mothers presenting with pruritus in pregnancy be routinely screened for the presence of ICP, hence identification of mothers with obstetric cholestasis and proper management adhering to guidelines will invariably reduce the fetal morbidity and mortality as well as maternal morbidity.

We also strongly recommend that facilities for diagnosis of ICP be implemented at least in the tertiary care state hospitals in the country.

\section{Limitations}

The cost of single assay of serum bile acids and other relevant investigations was the main limiting factor. Hence, we had to select the mothers antenatally presenting with pruritis in pregnancy. It has been also observed that some mothers on realizing that they had increased serum bile acids were requesting early delivery by elective caesarean section. However, these problems were dealt with close USS fetal monitoring and reassuring the mothers.

\section{Acknowledgements}

Our sincere thanks and appreciation are extended to the following institutions and individuals for their assistance towards success of the research: medical officers, nurses and staff at medical clinic, University Obstetrics and Gynaecology Unit, De Soysa Hospital for Women (DSHW), Colombo, Sri Lanka. Our sincere thanks also extended to Nawaloka Hospital PLC, patients and their families. We confirm the authenticity of this clinical research work and certify that these data have not been published elsewhere nor are currently under consideration for publication elsewhere. With the submission of this manuscript we would like to undertake that all authors of this research paper have directly participated in the planning, execution or analysis of this study; all authors of this paper have read and approved the final version submitted. 


\section{Declaration of Conflicting Interests}

The author(s) declared no potential conflicts of interest with respect to the research, authorship, and/or publication of this article.

\section{Guarantor}

Professor S. H. Dodampahala.

\section{References}

[1] (2011) Obstetric Choleatasis, Green-Top guidelines by Royal College of Obstetrics and Gynaecology, No. 43.

[2] Bacq, Y., Sapey, T., Brechot, M.C., Pierre, F., Fignon, A. and Dubois, F. (1997) Intrahepatic Cholestasis of Pregnancy: A French Prospective Study. Hepatology, 26, 358-364.

[3] Meng, L.J., Reyes, H., Axelson, M., et al. (1997) Progesterone Metabolites and Bile Acids in Serum of Patients with Intrahepatic Cholestasis of Pregnancy: Effect of Ursodeoxycholic Acid Therapy. Hepatology, 26, 1573-1579.

[4] Reyes, H. and Simon, F.R. (1993) Intrahepatic Cholestasis of Pregnancy: An Estrogen-Related Disease. Seminars in Liver Disease, 13, 289-301.

[5] Fisk, N.M. and Storey, G.N. (1988) Fetal Outcome in Obstetric Cholestasis. British Journal of Obstetrics and Gynaecology, 95, 1137-1143.

[6] Glantz, A., Marschall, H.-U. and Mattsson, L.-Å. (2004) Intrahepatic Cholestasis of Pregnancy: Relationships between Bile Acid Levels and Fetal Complication Rates. Hepatology [Internet], 40, 467-474. http://dx.doi.org/10.1002/hep.20336

[7] Laatikainen, T. and Tulenheimo, A. (1984) Maternal Serum Bile Acid Levels and Fetal Distress in Cholestasis of Pregnancy. International Journal of Gynecology \& Obstetrics, 22, 91-94.

[8] Serrano, M.A., Macias, R.I., Vallejo, M., et al. (2003) Effect of Ursodeoxycholic Acid on the Impairment Induced by Maternal Cholestasis in the Rat Placenta-Maternal Liver Tandem Excretory Pathway. Journal of Pharmacology and Experimental Therapeutics, 305, 515-524.

[9] Lu, J., Kuang, J. and Cheng, X. (2015) Study on the Relationship between Prenatal Monitoring Index in Intrahepatic Cholestasis of Pregnancy and Perinatal Prognosis. Zhonghua Liu Xing Bing Xue Za Zhi, 35, 1281-1283.

[10] Williamson, C. and Geenes, V. (2014) Intrahepatic Cholestasis of Pregnancy. Obstetrics \& Gynecology, 124, $120-133$.

[11] Kowalski, A., Janosz-Galdys, I., Olejek, A. and Bodzek, P. (2014) Correlation between Serum Levels of Bile Acids in Pregnant Women with Intrahepatic Cholestasis of Pregnancy and Condition of Their Newborns. Ginekologia Polska, 85, 101-104.

[12] Kowalska-Kanka, A., Maciejewski, T. and Niemiec, K.T. (2013) The Concentrations of Bile Acids and Erythropoietin in Pregnant Women with Intrahepatic Cholestasis and the State of the Fetus and Newborn. Medycyna Wieku Rozwojowego, 17, 232-245.

[13] Roncaglia, N., Arreghini, A., Locatelli, A., Bellini, P., Andreotti, C. and Ghidini, A. (2002) Obstetric Cholestasis: Outcome with Active Management. European Journal of Obstetrics \& Gynecology and Reproductive Biology, 100, 167-170.

[14] Puljic, A., Kim, E., Page, J., et al. (2015) The Risk of Infant and Fetal Death by Each Additional Week of Expectant Management in Intrahepatic Cholestasis of Pregnancy by Gestational Age. American Journal of Obstetrics \& Gynecology [Internet], 212, 667.e1-667.e5. http://dx.doi.org/10.1016/j.ajog.2015.02.012

[15] Brouwers, L., Koster, M.P.H., Page-Christiaens, G.C.M.L., et al. (2015) Intrahepatic Cholestasis of Pregnancy: Maternal and Fetal Outcomes Associated with Elevated Bile Acid Levels. American Journal of Obstetrics \& Gynecology [Internet], 212, 100.e1-100.e7. http://dx.doi.org/10.1016/j.ajog.2014.07.026

[16] Fisk, N.M., Bye, W.B. and Storey, G.N. (1988) Maternal Features of Obstetric Cholestasis: 20 Years Experience at King George V Hospital. Australian and New Zealand Journal of Obstetrics and Gynaecology, 28, 172-176.

[17] Heinonen, S. and Kirkinen, P. (1999) Pregnancy Outcome with Intrahepatic Cholestasis. Obstetrics \& Gynecology, 94, 189-193.

[18] Germain, A.M., Kato, S., Carvajal, J.A., Valenzuela, G.J., Valdes, G.L. and Glasinovic, J.C. (2003) Bile Acids Increase Response and Expression of Human Myometrial Oxytocin Receptor. American Journal of Obstetrics and Gynecology, 189, 577-582.

[19] Pusl, T. and Beuers, U. (2007) Intrahepatic Cholestasis of Pregnancy. Orphanet Journal of Rare Diseases [Internet], 2, 26. http://www.ncbi.nlm.nih.gov/pmc/articles/PMC1891276/ 
[20] Mullally, B.A. and Hansen, W.F. (2002) Intrahepatic Cholestasis of Pregnancy: Review of the Literature. Obstetrical \& Gynecological Survey, 57, 47-52.

[21] Reyes, H., Radrigan, M.E., Gonzalez, M.C., et al. (1987) Steatorrhea in Patients with Intrahepatic Cholestasis of Pregnancy. Gastroenterology, 93, 584-590.

[22] Sepulveda, W.H., Gonzalez, C., Cruz, M.A. and Rudolph, M.I. (1991) Vasoconstrictive Effect of Bile Acids on Isolated Human Placental Chorionic Veins. European Journal of Obstetrics \& Gynecology and Reproductive Biology, 42, 211-215.

[23] Zimmermann, P., Koskinen, J., Vaalamo, P. and Ranta, T. (1991) Doppler Umbilical Artery Velocimetry in Pregnancies Complicated by Intrahepatic Cholestasis. Journal of Perinatal Medicine, 19, 351-355. 